\title{
Refining Black Hole Physics to Obtain Planck's Constant from Information Shared from Cosmological Cycle to Cycle (Avoiding Super-Radiance)
}

\author{
Andrew Walcott Beckwith \\ Physics Department, College of Physics, Chongqing University, Chongqing, China \\ Email: Rwill9955b@gmail.com, abeckwith@uh.edu
}

How to cite this paper: Beckwith, A.W. (2019) Refining Black Hole Physics to Obtain Planck's Constant from Information Shared from Cosmological Cycle to Cycle (Avoiding Super-Radiance). Journal of High Energy Physics, Gravitation and Cosmology, 5, 464-472. https://doi.org/10.4236/jhepgc.2019.52027

Received: February 18, 2019

Accepted: April 6, 2019

Published: April 9, 2019

Copyright ( 2019 by author(s) and Scientific Research Publishing Inc. This work is licensed under the Creative Commons Attribution International License (CC BY 4.0).

http://creativecommons.org/licenses/by/4.0/

(c) (i) Open Access

\begin{abstract}
Padmanabhan elucidated the concept of super radiance in black hole physics which would lead to loss mass of a black hole, and loss of angular momentum due to space-time infall of material into a black hole. As Padmanabhan explained it, to avoid super radiance, and probable break down of black holes, from in fall, one would need in fall material frequency, divided by mass of particles undergoing in fall in the black hole to be greater than the angular velocity of the black hole event horizon in question. We should keep in mind we bring this model up to improve the chance that Penrose's conformal cyclic cosmology will allow for retention of enough information for preservation of Planck's constant from cycle to cycle, as a counterpart to what we view as unacceptable reliance upon the LQG quantum bounce and its tetrad structure to preserve memory. In addition, we are presuming that at the time of $z=20$ in red shift that there would be roughly about the same order of magnitude of entropy as number of operations in the electro weak era, and that the number of operations in the $z=20$ case is close to the entropy at redshift $z=0$. Finally, we have changed $\Lambda$ with the result that after redshift $=20$; there is a rapid collapse to the present-day vacuum energy value i.e. by $z=12$ the value of the cosmological constant, $\Lambda$ likely being the same, today, as for what it was when $z=12$. And $z=12$ is the redshift value about when Galaxies form.
\end{abstract}

\section{Keywords}

Planck's Constant, Black Hole, Super Radiance

\section{Introduction}

We start with the premise that LQG tetrad structure will in itself not be suffi- 
cient to preserve cosmological memory from cosmological cycle to cycle. Appendix A outlines how we view the well intentioned LQG memory preservation program and the alternative, a refinement of the conformal cyclic cosmology program of Penrose which will make use of refining the concept of super radiance and how to avoid it, so as to heighten the chance of preserving cosmological "memory" from one cycle of creation to another one of the candidates for memory transfer given by data as supplied by Natarajan in GR 20 in pre galactic black holes formed at about $z=20$ to $Z=12$ (red shift) times by super massive black holes at least 500 times the mass of our star, Sol. The candidate for information inflow into the initially massive black holes as we choses it would be manifest in relic gravitational waves. To quantify in fall into these primordial black holes we will represent GW by massive gravitons, with the mass of a graviton as given by

$$
-3 m_{\text {graviton }}^{2} \cdot h=\frac{\kappa}{2} \cdot T
$$

Our work uses Visser's [1] analysis of non-zero graviton mass for both $T$ and h.

Furthermore, his version of $g_{u v}=\eta_{u v}+h_{u v}$ can be written as setting

$$
h_{u v}=\frac{2 G M}{r} \cdot\left(\exp \left(\frac{m_{\text {graviton }} \cdot r}{\hbar}\right)\right) \cdot\left(2 V_{u} V_{v}+\eta_{u v}\right)
$$

If one adds in velocity "reduction" put in with regards to speed propagation of gravitons [1]

$$
v_{\text {graviton }}=c \cdot \sqrt{1-\frac{m_{\text {graviton }}^{2} \cdot c^{4}}{\hbar^{2} \omega_{\text {graviton }}^{2}}}
$$

We argue that in doing so that we are paying strict attention to the issues brought up in the following references [2] [3] and [4] which the author has discussed in Rencontres De Moriond, in 2017, in the cosmology section, as of March 2017, and which came up also in Dice 2018, September, and also in Marcel Grossman 15 in innumerable discussion the author had in July 2018 in the Black hole symposium run by Alexander Zakharov, in Rome Italy in his 3 days of Black hole physics which the author partook of as a Poster entry in that part of the Marcel Grossman 15 conference.

One can insert all this into Equation (1) to obtain a real value for the square of frequency $>0$, i.e. note that Kim's article [5] is with regards to Gravitons in brane/string theory, but it is likely that the same dynamic for semi classical representations of a graviton with mass.

\section{Conditions Allowing for Recycling of Planck's Constant in Penrose's Cyclic Universe Model Revisited}

The main methodology in the Penrose proposal has been in Equation (4) evaluating a change in the metric $g_{a b}$ by a conformal mapping $\widehat{\Omega}$ to

$$
\hat{g}_{a b}=\widehat{\Omega}^{2} \cdot g_{a b}
$$


Penrose's suggestion has been to utilize the following

$$
\widehat{\Omega} \underset{c c c}{\longrightarrow} \widehat{\Omega}^{-1}
$$

Infill into cosmic black hopes has been the main mechanism which the author asserts would be useful for the recycling apparent in Equation (5) above with the caveat that $\hbar$ is kept constant from cycle to cycle as represented by

$$
\hbar_{\text {old cosmology cycle }} \equiv \hbar_{\text {present cosmology cycle }}
$$

What would be crucial in doing both Equation (5) and Equation (6) would be in specifying how massive black holes as of at least 500 times the mass of the sun, i.e. $500 M_{\odot}$, large mass of a black hole formed between $Z=20$ and $Z=12$ redshifts i.e. before galaxy formation, would tend toward conditions for which Equation (6) could be fulfilled. We do this by looking at details for $\widehat{\Omega}$ satisfied by information gathering which we bring up now.

\section{Necessary Construction Details for the Mapping $\Omega$}

The procedures for giving linkage to a formulation of $\hat{\Omega}$ means that one must consider a basic construction given by Penrose as to his ccc proposal. Note also [6]. And the Penrose ccc proposal which we will outline below, namely that one has

$$
\begin{aligned}
& E=8 \pi \cdot T+\Lambda \cdot g=\text { Source for gravitational field } \\
& T=\text { mass energy density term } \\
& g=\text { gravitational metric } \\
& \Lambda=\text { vacuum energy }
\end{aligned}
$$

with

$$
\Lambda=c_{1} \cdot(\text { Temp })^{\beta}
$$

For an invariant $E$ for cycle to cycle no matter what Equation (8) gives us, this leads to the following statement as to a formulation of $\hat{\Omega}$ i.e.

$$
\begin{aligned}
& \left.E\right|_{\text {initial }}=\left.\left.\left.E\right|_{\text {final }} \Leftrightarrow \Lambda \cdot g\right|_{\text {initial }} \equiv \Lambda \cdot g\right|_{\text {final }} \\
& \left.\left.\& \Lambda \cdot g\right|_{a b}\right|_{\text {final }}=\left(\left.\Lambda\right|_{\text {final }}\right) \cdot \widehat{\Omega}^{2} \cdot\left(\left.g_{a b}\right|_{\text {initial }}\right)
\end{aligned}
$$

i.e. is this possible? only if there are very small initial wavelengths at/before the electro weak regime, i.e. a wave length perhaps as small as Planck length. The problem is that in doing so, and this appears to be intuitive and obvious, something which is contradicted by Durrer's [7] treatment of early universe plasma waves generating early universe GW. We then state for hypothesis that if $N$ is a numerical count which has led us to hypothesize using

$$
\widehat{\Omega} \propto S_{\text {entropy }} \approx N
$$

\section{Information as Given in Space-Time Infall into Early Universe Black Holes and the Problem of Avoiding Super-Radiance}

Note that Beckwith [8] has used Y. Ng's [9] counting algorithm with regards to 
entropy, and non-zero mass (massive) gravitons, where

$$
S \approx N \cdot\left(\log \left[V / \lambda^{3}\right]+5 / 2\right) \approx N
$$

Furthermore, making an initial count of gravitons with $S \approx N \sim 10^{7}$ gravitons with Seth Lloyd's [10] $I=S_{\text {total }} / k_{B} \ln 2=[\text { \# operations }]^{3 / 4} \sim 10^{7}$ as implying at least one operation per unit graviton, with gravitons being one unit of information, per produced graviton. Note, Smoot [11] gave initial values of the operations as

$$
\text { [\# operations }]_{\text {initially }} \sim 10^{10}
$$

The number of operations, if tied into bits of "information" may allow for space time linkages of the following value of the fine structure constant, as from a prior to a present universe, once initial conditions of inflation may be examined experimentally, i.e. looking at inputs into [8], i.e. the fine structure constant given in [8], which has presumably the value of

$$
\tilde{\alpha} \equiv \frac{e^{2}}{\hbar \cdot c} \equiv \frac{e^{2}}{d} \times \frac{\lambda}{h \cdot c}
$$

As of the electroweak era

$$
\begin{aligned}
& \left.\left.\left.S_{\text {entropy }}\right|_{\text {ew }} \sim N \sim 10^{51}\right|_{\text {ew }} \propto[\text { \# operations }]^{3 / 4}\right|_{\text {ew }} \\
& \left.\Leftrightarrow[\text { \# operations }]\right|_{\text {ew }} \approx 10^{71}
\end{aligned}
$$

We are presuming that at the time of $z=20$ in red shift that there would be roughly about the same order of magnitude of entropy as number of operations in the electro weak era, and that the number of operations in the $z=20$ case is close to the entropy at redshift $z=0$

$$
\begin{aligned}
& \left.\left.\left.S_{\text {entropy }}\right|_{\text {redshift=20 }} \sim N \sim 10^{67}\right|_{\text {redshift=20 }} \propto[\text { \# operations }]^{3 / 4}\right|_{\text {redshift=20 }} \\
& \left.\Leftrightarrow[\# \text { operations }]\right|_{\text {redshift=20 }} \approx 10^{89}
\end{aligned}
$$

After this is done it is useful to note that the number of operations as to the redshift $=20$ at the formation of the first set of super massive black holes would be about the same as entropy today which the author views as no accident i.e. the number of super massive black holes is at least 100,000 or more with a mass of at least one hundred to ten thousand times the mass of the sun

Then there could be an infall of less than $10^{84}-10^{87}$ operations/per black bole. After this is done, note that the particle in fall per black hole is less than $10^{62}$ value. Interstellar space has 2.73 Kelvin and was only semi hotter at a red shift at $z=20$. The situation then is that a black hole five times the mass of the sun would have a temperature $12 \times 10^{-9}$ Kelvin, and that could easily drop to about $10^{-11}$ Kelvin, which is about the temperature for a black hole 20 - 50 times the mass of the sun, and that due to

$$
T_{\text {Black hole }}=\frac{\hbar c^{3}}{8 \pi k G M_{\text {Black hole }}}
$$


Then the next step would be to look at the resulting temperature differential flowing into the black hole which would be

$$
\left.\Delta T\right|_{\text {near Black hole }}=T_{\text {background }}-T_{\text {Black hole }} \approx 10^{\alpha} \text { Kelvin } \geq 2.73 \text { Kelvin }
$$

We look at a black hole of would be mass $10^{22} \mathrm{~kg} \propto 10^{58} \mathrm{eV} / \mathrm{c}^{2}$, mass of this value would be for a black hole having the temperature given in Equation (15), leading to if each graviton having $10^{-29} \mathrm{eV} / \mathrm{c}^{2}$ number of gravitons of about $10^{87}$ gravitons; then leading to for 1000 super massive black holes an intake of approximately $10^{90}$ later entropy-value into $100-1000$ black holes. This is equivalent to processes coming from infall from cosmological space-time into 100 1000 black holes. This is close to the present value of entropy today and is similar to values given by Lloyd as to the entropy of the present universe, which we do not think is an accident. Therefore, the inflow of heat into $100-1000$ pre galactic black holes which is a thermal energy, as given by 100 - 1000 super massive early black holes is equivalent to $10^{90}$ later entropy equivalent. Also the value of about $10^{84}-10^{87}$ operations/per black bole, we look at less than or equal values of the number of operations set up to be processed as given by the electroweak era to be eventually generating approximately a $10^{90}$ later entropy numerical count as created about $z=20$, which again we think is no accident.

\section{A Way to Create Conditions for Planck Value from Cycle to Cycle, While Tackling the Problem of Super Radiance and Black Hole Physics as of about $z=20$ and Making Sense of $\widehat{\Omega}$}

To do this note that $\hat{\Omega}$ we write as proportional to entropy, specifically because of; $\left.\Lambda \cdot g_{a b}\right|_{\text {final }}=\left(\left.\Lambda\right|_{\text {final }}\right) \cdot \widehat{\Omega}^{2} \cdot\left(\left.g_{a b}\right|_{\text {initial }}\right)$ we write entropy as given by Cai as, if $S \propto r_{\oplus}$ and $r_{\oplus}$ is the radius of the black hole horizon, then if $\mathrm{M}$ is the mass of a black hole

$$
S_{\text {entropy }} \leq N=\frac{3 G}{G \Lambda} \Leftrightarrow S_{\text {Black hole }} \approx M_{\text {Black hole }}^{2}
$$

Also for the angular velocity, as given by $\Omega_{H} \propto 1 / r_{\oplus}$

$$
\Omega_{H} \propto 1 / r_{\oplus} \propto 1 / \sqrt{S_{\text {Black hole }}} \propto 1 / \sqrt{N} \propto 1 / \sqrt{(\text { Temp })^{\beta}}
$$

We write the angular velocity as intertwined into Padmanabhlan's description of super radiance by noting that for infall into a black hole creating instability and loss of angular momentum for the black hole we would have super radiance for frequency and for mass of an infalling particle $\tilde{m}$ into a black hole

$$
0<\left(\omega_{\text {particles }} / \tilde{m}\right)<\Omega_{H}
$$

To avoid super radiance, we would have, conversely

$$
\left(\omega_{\text {particles }} / \tilde{m}\right)>\Omega_{H}
$$

Or 


$$
\omega_{\text {particles }}>\tilde{m} / \sqrt{N}
$$

The frequency of incoming particles allowing for stable black holes would be extremely low, i.e. almost any gravitational radiation and graviton infall into Penrose mandated black holes would do it. If one looks at the contribution of four and five dimensional black holes to entropy, with $L$ being the dimension of a fifth dimension we obtain

$$
\left.S_{\text {Black hole }}\right|_{4 \mathrm{D}}=4 \pi M_{\text {Black hole }}^{2}
$$

And

$$
\left.S_{\text {Black hole }}\right|_{5 \mathrm{D}}=4 \pi M_{\text {Black hole }}^{2} \cdot \sqrt{8 L / 27 \pi M_{\text {Black hole }}}
$$

The outcome would be to have for five dimensional black holes no super radiance if

$$
\left.\omega_{\text {particles }}\right|_{5 \mathrm{D}}>(\tilde{m} / \sqrt{N}) \cdot\left(27 \pi M_{\text {Black hole }} / 8 L\right)^{1 / 4}
$$

The frequency goes down as $L$ increases in size.

\section{Black Hole Physics as of about $z=20$ and Making Sense of $\hat{\Omega}$}

We also can write

$$
\left.\omega_{\text {particles }}\right|_{5 \mathrm{D}}>(\tilde{m} / \sqrt{\Omega}) \cdot\left(27 \pi M_{\text {Black hole }} / 8 L\right)^{1 / 4}
$$

From $\hat{\Omega} \sim 3 \pi G^{-1} / \Lambda$ and $\Lambda=c_{1} \cdot(\text { Temp })^{\beta}$, and making use of the relations as given by $T_{\text {Black hole }}=\frac{\hbar c^{3}}{8 \pi k G M_{\text {Black hole }}},\left.\Delta T\right|_{\text {near Black hole }}=T_{\text {background }}-T_{\text {Black hole }}$ due to $\hbar$ in the relations we are able to ascertain per black hole

$$
\left.\hbar_{\text {per black hole }} \propto[\Delta T \text { (due to background per black hole })\right] \cdot\left(8 \pi k G M_{\text {Black hole }} / c^{3}\right) \text { (26) }
$$

Also if at $z=20$ there is the number of $N_{z=80 B H}$ black holes at $z=20$, the contribution to $\hbar_{\text {old cosmology cycle }} \equiv \hbar_{\text {present cosmology cycle }}$ then can be said to be

$$
\begin{aligned}
& \hbar_{z=20} \propto\left(N_{z=20} \text { Black holes }\right) \\
& \text { times }[\Delta T \text { (due to background per black hole })] \cdot\left(8 \pi k G M_{\text {Black hole }} / c^{3}\right)
\end{aligned}
$$

We also look then at the value of $\widehat{\Omega}$ at $z=20$ and get from Equation (27)

$$
\left.\widehat{\Omega}_{\text {Redshift } z=20} \propto N \approx 10^{67}\right|_{\text {Redshift } z=20}
$$

And we keep in mind that

$$
\begin{aligned}
& \left.\left(\left.\widehat{\Omega}_{\text {Redshift } z=20} \propto N \approx 10^{67}\right|_{\text {Redshift } z=20}\right) \cdot \Lambda\right|_{\text {Redshift } z=20} \\
& \left.\approx\left(\left.\widehat{\Omega}_{\text {Redshift } z=20} \propto N \approx 10^{89}\right|_{\text {Redshift } z=20}\right) \cdot \Lambda\right|_{\text {Redshift } z=20}
\end{aligned}
$$

The above hypothesizes changing $\Lambda$ with the result that after redshift $=20$ there is a rapid collapse to the present day vacuum energy value i.e. by $z=12$ we have that $\Lambda$ likely the same as today which would be about when Galaxies form. 


\section{Acknowledgements}

This work is supported in part by National Nature Science Foundation of China grant No. 11375279.

\section{Conflicts of Interest}

The author declares no conflicts of interest regarding the publication of this paper.

\section{References}

[1] Visser, M. http://arxiv.org/pdf/gr-qc/9705051v2

[2] Lavenda, B.H. and Dunning-Davies, J. (1990) Qualms Concerning the Inflationary Scenario. Foundation of Physics Letters, 5, 191-196. http://milesmathis.com/dunning.pdf

[3] Tolman, R.C. (1934) Relativity and Cosmology. Clarion Press, Oxford.

[4] Maggiore, M. (2008) Gravitational Waves, Volume 1: Theory and Experiment. Oxford Univ. Press, Oxford.

[5] Kim, J.Y. http://arxiv.org/pdf/hep-th/0109192v3

[6] Beckwith, A. http://vixra.org/abs/1006.0027

[7] Durrer, R. and Rinaldi, M. (2009) Graviton Production in Non-Inflationary Cosmology. Physical Review D, 79, Article ID: 063507. http://arxiv.org/abs/0901.0650

[8] Beckwith, A.W. (2019) A Kaluza Klein Treatment of a Graviton and Deceleration Parameter $\mathrm{Q}(\mathrm{Z})$ as an Alternative to Standard DE and Its Possible Link to Standard DE Equation of State as Given by Li, Li, Wang and Wang, in 2017. Journal of High Energy Physics, Gravitation and Cosmology, 5, 208-217. https://doi.org/10.4236/jhepgc.2019.51012

[9] Ng, Y. (2008) Spacetime Foam: From Entropy and Holography to Infinite Statistics and Nonlocality. Entropy, 10, 441-461. https://doi.org/10.3390/e10040441

[10] Lloyd, S. (2002) Computational Capacity of the Universe. Physical Review Letters, 88, Article ID: 237901.

[11] Smoot, G. http://chalonge.obspm.fr/Paris07_Smoot.pdf

[12] Ellis, G., Maartens, R. and MacCallum, M.A.H. (2012) Relativistic Cosmology. Cambridge University Press, Cambridge.

[13] Beckwith, A.W. (2019) How $(\Delta t) 5+A_{1}(\Delta t)^{2}+A_{2}=1$ Is Generally, in the Galois Sense Solvable for a Kerr-Newman Black Hole Affect Questions on the Opening and Closing of a Wormhole Throat and the Simplification of the Problem, Dramatically Speaking, If $d=1$ (Kaluza Klein Theory) and Explaining the Lack of Overlap with the Results When Applying the Gauss-Lucas Theorem. Journal of High Energy Physics, Gravitation and Cosmology, 5, 235-278. https://doi.org/10.4236/jhepgc.2019.51014

[14] Scully, S. (2017) Semiclassical Gravity: A Testable Theory of Quantum Gravity. In: Hossfelder, S., Ed., Experimental Search for Quantum Gravity, Springer FIAS interdisciplinary Science Series, Springer Verlag, Frankfurt, 69-76.

[15] Frassino, A.M. (2017) Quantum Gravity Deformations. In: Hossfelder, S., Ed., EXperimental Search for Quantum Gravity, Springer FIAS Interdisciplinary Science Series, Springer Verlag, Frankfurt, 77-83. 
[16] Bohmer, C.G. (2017) Introduction to General Relativity and Cosmology. World Scientific, Singapore.

[17] Giovannini, M. (2008) A Primer on the Physics of the Cosmic Microwave Background. World Scientific, Singapore.

[18] Abbott, B.P., et al. (2016) Observation of Gravitational Waves from a Binary Black Hole Merger. Physical Review Letters, 116, Article ID: 061102.

[19] Abbott, B.P., et al. (2016) GW151226: Observation of Gravitational Waves from a 22-Solar-Mass Binary Black Hole Coalescence. Physical Review Letters, 116, Article ID: 241103.

[20] Abbott, B.P., et al. (2017) GW170104: Observation of a 50-Solar-Mass Binary Black Hole Coalescence at Redshift 0.2. Physical Review Letters, 118, Article ID: 221101.

[21] Corda, C. (2009) Interferometric Detection of Gravitational Waves: The Definitive Test for General Relativity. International Journal of Modern Physics D, 18, 2275-2282. https://arxiv.org/abs/0905.2502 


\section{Appendix A}

\section{The generalized Stress Energy component of GR considered which part we evaluate.}

To do this we look at, from [12] a GR Einstein stress energy tensor we write as, with $u_{a}$ the four vector velocity. Also, $\rho$ is the relativistic energy density, $q_{a}$ the relativistic momentum density, and $p$ is pressure, and $\pi_{a b}$ the relativistic anisotropic stress tensor due to viscosity, magnetic fields. $\rho$ has a gravitational radiation component. Effectively, Equation (A1) has $\rho=\rho_{\mathrm{GW}}+\rho_{\text {everything else }}$ such that

$$
\begin{aligned}
& T_{a b}=\rho u_{a} u_{b}+q_{a} u_{b}+u_{a} q_{b} p h_{a b}+\pi_{a b} \\
& \Leftrightarrow T_{a b}=T_{\text {GW/Gravtitions }}+T_{\text {everything else }}
\end{aligned}
$$

We further state, that if we have blackholes as interconnected with worm holes, as given in [13] that what is given in Equation (A1) above has counter parts in a necessary condition for quantization which can be seen in [14] for what is known as semi classical gravity. If we can make a semi classical approximation, then the energy as given by the zeroth order approximation may be indeed treatable by the Schrodinger-Newton equation as in [14] which leads us to consider the Equation (6) and Equation (7) of reference [14]. On the other hand, if what we bring up in [13] is mainly applicable, we then will take the zeroth order approximation of Equation (A1) and use what is in [15], as far as a multi-dimensional generalization of the Heisenberg Uncertainty principle, as given in [15]. This again will necessitate refinement of what is called in [16], pages 117-123 the physics of the so called "interior solutions of the Schwarzschild Metric" which the author recommends people review, with an idea toward answering if the energy component of the stress energy tensor could possibly be related to Uncertainty principle physics, or something else. We close then with a recommendation that the reviewers of this paper also look at [17], especially pages 141 to 151 to ascertain how our work may give insight as to the possibility of quantum mechanical conditions which could give quantum description of tensor modes which would relate to gravitational wave physics. This in turn may enable experimental inquiry tying in what we are modeling with the physics given in references [18] [19] [20] [21]. 